\title{
Patient perspectives on Parkinson's disease therapy in Japan and the United States: results of two patient surveys
}

This article was published in the following Dove Press journal:

Patient Related Outcome Measures

10 July 2012

Number of times this article has been viewed

\section{Nobutaka Hattori' \\ Kenichi Fujimoto ${ }^{2}$ \\ Tomoyoshi Kondo 3 \\ Miho Murata ${ }^{4}$ \\ Mark Stacy ${ }^{5}$}

'Department of Neurology, Juntendo

University School of Medicine,

Tokyo; ${ }^{2}$ Department of Neurology,

Jichi Medical University, Tochigi;

${ }^{3}$ Department of Neurology, Wakayama

Medical University, Wakayama;

${ }^{4}$ Department of Neurology, National

Center Hospital of Neurology

and Psychiatry, Tokyo, Japan;

${ }^{5}$ Division of Neurology, Duke

University, Durham, NC, USA
Correspondence: Nobutaka Hattori

Department of Neurology,

Juntendo University School

of Medicine, 2-I-I Hongo, Bunkyo-ku,

Tokyo, I I3-842I, Japan

$\mathrm{Tel}+8|338| 33|| \mid$

Fax +81338149100

Email nhattori@juntendo.ac.jp
Background: Despite evidence suggesting that patient attitudes towards therapy may influence treatment outcomes, the impact of these factors on treatment for Parkinson's disease is poorly understood. These two surveys, based in Japan and the US, investigated the attitudes of patients towards antiparkinsonian medications, the complications of these therapies, and how these differ across geographies.

Methods: The US PRELUDE survey collected data from May 13 to May 20, 2003, from 300 interviews with patients with Parkinson's disease from the National Parkinson Foundation. The Japanese survey was carried out from June to December 2008 in a stepwise manner using questionnaires $(n=3548)$ followed by interviews with those who had consented to participate in the questionnaire $(n=407)$. Both surveys assessed the attitudes of patients towards therapies for Parkinson's disease and associated complications.

Results: Dyskinesia was not a major challenge of therapy for Parkinson's disease, and wearingoff caused greater concern in the US, while hallucinations had a greater emphasis in Japan. Patients who had previously experienced dyskinesia were less concerned about this side effect than those who had not. Although pill burden was thought to be a concern in the US, Japanese patients did not indicate that pill burden would limit their drug intake. There were also discrepancies between the perspectives and concerns of patients and those of their treating physicians.

Conclusion: Recognizing patient perspectives regarding therapies for Parkinson's disease and associated complications, as well as certain cultural influences, is important in the management of parkinsonian symptoms. Acknowledging these concerns may improve the standard of care in patients with Parkinson's disease. In addition, improved patient education and effective patient-physician communication in both countries may improve compliance and treatment outcomes in patients with the disease.

Keywords: Parkinson's disease, patient concerns, dyskinesia, wearing-off, hallucinations

\section{Introduction}

It is generally accepted that patient health and therapeutic outcomes are influenced by beliefs about and attitudes toward medications, and expectations from therapy, as well as level of education and awareness about the disease and its management. ${ }^{1,2}$ This is particularly true for long-term, chronic illnesses, whereby patients must make lifestyle adjustments to accommodate increasing disability. ${ }^{2,3}$ Patient decisions to follow a recommended treatment are also likely to be influenced by beliefs about medications and understanding about a medical illness. For example, despite the prevalence of available therapies, there is a high rate of early treatment discontinuation in patients suffering from depression, owing to factors such as a perceived stigma of mental 
health problems, which consequently impacts therapeutic outcome. ${ }^{4}$ Although it is evident that these factors play a role in treatment outcomes, the influence of patient perspectives towards therapy has not been well documented.

Patient attitudes regarding Parkinson's disease (PD) may influence the types and dosing frequencies of medications available for symptomatic treatment. PD is a progressive, chronic illness that impacts motor abilities and quality of life. The armamentarium for PD management includes many agents that are associated with a wide range of benefits and potential risks. For example, levodopa is associated with an increased risk of motor complications, including dyskinesia and motor fluctuation (wearing-off), while the side effects of dopamine agonists include hallucinations, somnolence, edema, and impulse control disorders., ${ }^{5,6}$ Tailoring therapy according to individual unique symptoms is important to achieve successful treatment outcomes. ${ }^{5}$ Patient perspectives on treatment strategies, and the differences in these factors across different geographies, are poorly defined. Understanding these differences may improve therapeutic outcomes.

To this end, two surveys were conducted, one in the US and another in Japan, to investigate the attitudes and concerns of patients regarding PD therapy. The results of these surveys suggest unmet needs regarding PD therapy, as well as discrepancies between patient and physician perspectives. They also identify cultural differences in patient attitudes.

\section{Materials and methods}

PRELUDE (PRoject to Examine Levodopa Utilization DEcisions) was a two-part survey carried out in the US, comprising patient and physician questionnaires.

\section{Patient survey}

Data were collected from May 13 to May 20, 2003, from 300 interviews of people with PD currently using levodopa-carbidopa therapy. The respondents were sampled from the National Parkinson Foundation list of 10,000 email newsletter recipients; invitations to participate in the survey were embedded in the National Parkinson Foundation email newsletter and sent each day until all 300 questionnaires were completed. Each respondent was assigned an individual identification number and password to ensure that patients only completed the survey once. For each participant, a US \$15 honorarium for completing the study and a US \$15 donation to the National Parkinson Foundation were given.

\section{Physician survey}

In this part of the survey, data were collected online between April 16 and 29, 2003, from 328 general neurologists, 74 movement disorder specialists, and 54 primary care physicians. To qualify, all physicians must have treated patients with PD (at least some with levodopa-carbidopa) and have been in practice for $\geq 2$ years and $\leq 30$ years.

\section{Japanese survey}

This survey focused on patient attitudes toward PD and its treatment, and was completed in Japan from June to December 2008 in a stepwise manner, initially with questionnaires, then interviews with those who had consented in the questionnaire to be interviewed.

Questionnaires were sent to approximately 7000 members of the Japan Parkinson Disease Association and about 1200 nonmembers. Data were collected from 4011 respondents between July and August 2008. A total of 387 participants who received deep brain stimulation were excluded, and 3548 evaluable respondents were assessed. A total of 2316 of these patients provided their consent to participate in interview-based research. Patients were extracted at random.

A total of 407 of the patients who responded to the questionnaire-based survey participated in the on-site, interview-based survey between August and December 2008. Thirty-six participants who received deep brain stimulation were excluded from the analysis relating to drug medication.

The presence of wearing-off or dyskinesia was determined in both parts of the survey. In the questionnaire-based survey, this was accomplished by enquiring about the efficacy of medication. During the interview part of the survey, patients were asked to record the severity of symptoms in relation to timing of each dose in a diary for one day prior to the interview. Symptom severity was based on patient self-perception and was measured using the Hoehn and Yahr scale by examining physicians.

\section{Results}

\section{Patient characteristics}

Patient characteristics for both the Japanese and US PRELUDE studies are summarized in Table 1. Patients with potential motor fluctuations and several years of treated PD were recruited in both surveys. In Japan, 95\% of patients were receiving levodopa, mostly as therapy supplementary to dopamine agonists or monoamine oxidase B inhibitors. Between $70 \%$ and $80 \%$ of the US PRELUDE respondents were receiving levodopa therapy; around half of these 
Table I Patient characteristics

\begin{tabular}{lll}
\hline Patient characteristics & Japanese study & US study \\
\hline Mean age (years) & 69 & N/A \\
Duration of PD (years) & $3-9$ (majority) & 7 (mean) \\
Patients receiving levodopa (\%) & 95 & $70-80$ \\
Patients receiving DAs (\%) & 85 & 57 \\
Patients receiving MAO-B & 42 & N/A \\
inhibitors (\%) & & \\
$\begin{array}{l}\text { Patients receiving COMT } \\
\text { inhibitors, including }\end{array}$ & 25 & 29 \\
entacapone (\%) & & \\
\hline
\end{tabular}

Abbreviations: PD, Parkinson's disease; DA, dopamine agonists; MAO-B monoamine oxidase B; N/A, not applicable; COMT, catechol-O-methyl transferase.

received dopamine agonist treatment before initiating levodopa-carbidopa therapy.

\section{Attitude towards motor complications and other adverse effects \\ Wearing-off is a concern for PD patients}

In the US PRELUDE study, more PD patients were concerned about wearing-off (55\%) than about dyskinesia (23\%, Figure 1). Although primary care physicians generally agreed with this concern (63\%), specialists considered dyskinesia to be a greater concern $(32 \%$ of neurologists and $50 \%$ of movement disorder specialists, compared with $7 \%$ of primary care physicians).

\section{Balance between adverse effects and efficacy of medication}

In Japan, more than half of patients experiencing fluctuations preferred to avoid the adverse effects of antiparkinsonian medication rather than obtain effective relief from bradykinesia (Figure 2). However, the number of patients preferring relief from bradykinesia gradually increased with escalating symptom severity during off periods. This preference for avoiding adverse effects was similar in patients with (47.6\%) and without (47.9) wearing-off. When interviewed, Japanese patients who preferred to avoid adverse effects were more concerned about hallucinations ( $44.6 \%$ of unaided responses) than other adverse effects such as constipation (12.2\%), drowsiness $(9.5 \%)$, or nausea $(6.8 \%)$. In fact, compared with hallucinations, dyskinesia was identified as an important adverse effect by fewer Japanese patients (Figure 3).

\section{Balance between dyskinesia and efficacy of medication}

In the overall population, Japanese patients experiencing on/off fluctuations preferred to avoid dyskinesia (about 45\%) rather than achieve relief from bradykinesia (about 25\%). Patients who had not yet experienced dyskinesia were more keen to avoid this complication (approximately 55\%) rather than obtain relief from bradykinesia (approximately 45\%), whereas patients who had already developed dyskinesia were less concerned about this adverse effect (approximately 40\%). Reasons for this concern in patients who had not yet experienced dyskinesia included anticipation of the mental burden of this adverse effect from observing it in other patients. They were also concerned that dyskinesia might prevent them from carrying out normal daily activities, such as working, and were worried about others' reactions to these abnormal movements when in public. In contrast, patients with mild dyskinesia tended to prefer improved mobility versus avoiding dyskinesia (nearly $42 \%$ versus $32 \%$ ). However, patients who had experienced severe dyskinesia indicated they would rather avoid this adverse effect (about 42\%) than obtain relief from bradykinesia (around 37\%).

\section{Patient attitudes towards medication}

In the US, patients' main concerns about levodopa-carbidopa were wearing-off, long-term side effects of levodopa-carbidopa therapy, and disease progression (Table 2). Although generally

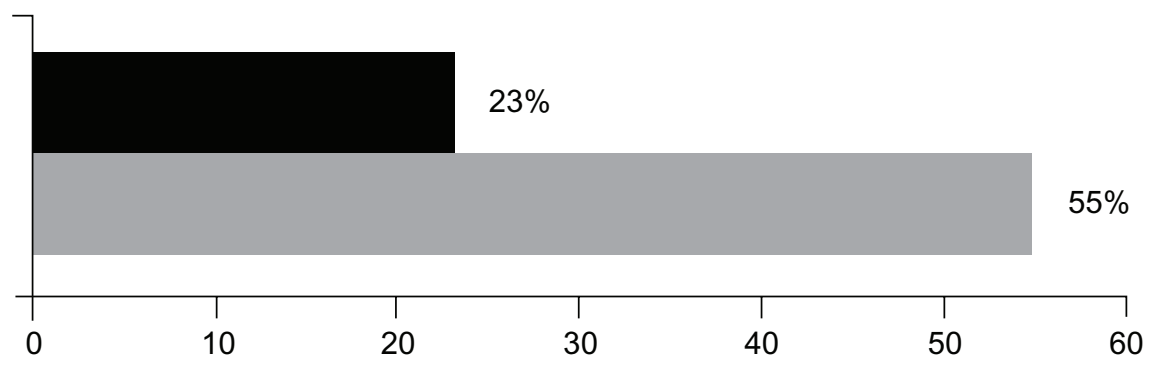

(\%)

Dyskinesias

Wearing-off

Figure I Percentage of patients in the US survey reporting dyskinesias or wearing-off as the greatest challenge of therapy. 


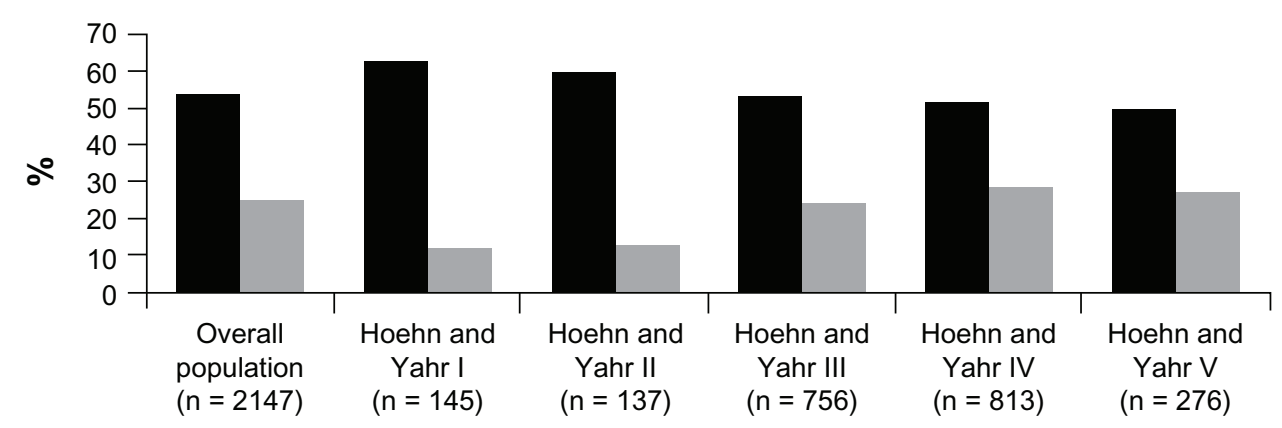

Patients who prefer to avoid AEs

Patients who prefer relief from bradykinesia

Figure 2 Percentage of patients in the Japanese survey who preferred to avoid adverse events compared with those who preferred relief from bradykinesia. Note: Hoehn and $\mathrm{Yahr}^{7}$ measurements were taken during off periods.

Abbreviation: $A E$, adverse effect.

satisfied with levodopa-carbidopa, 55\% of US patients with PD were at least somewhat concerned about taking levodopa-carbidopa, mostly owing to information gathered from the Internet $(69 \%)$ or from varied sources $(58 \%)$. In addition, $26 \%-34 \%$ of patients indicated that their concern stemmed from information from physicians, support groups, or newsletters.

In Japan, the percentage of patients dissatisfied with current pharmacotherapy tended to increase with a longer duration of PD. In addition, patients who were not suffering from wearing-off were more satisfied with their current pharmacotherapy than those who were experiencing wearing-off symptoms ( $49 \%$ versus $36 \%$, respectively).

\section{Attitudes towards drug intake and dose increases}

In the US, physicians felt strongly that patients would be more satisfied with reduced pill burden $(96.3 \%)$. They also believed that drug dissatisfaction stemmed from inconsistencies in symptom control achieved with generic formulations of levodopa-carbidopa (62.6\%).

When interviewed, Japanese patients generally preferred to obtain relief from bradykinesia (about 50\%) rather than limit their medication intake (about 40\%). This preference for symptomatic relief was similar in patients with or without wearing-off. The main reason for this preference was a desire to carry on with day-to-day activities, such as employment or housework. Patients who preferred to limit drug intake and dose were concerned about the adverse effects associated with increased pharmacotherapy $(68.5 \%)$; concern about wearing-off accounted for $10.5 \%$ of unaided responses. Other reasons for preferring to limit dose intake included compliance $(4.8 \%)$ and the apprehension that drugs may affect health $(6.5 \%)$.

Interestingly, the levodopa-equivalent dose of antiparkinsonian medication did not differ between satisfied and dissatisfied patients in Japan. According to a survey of 121 Japan-based physicians at the 2008 Movement Disorders

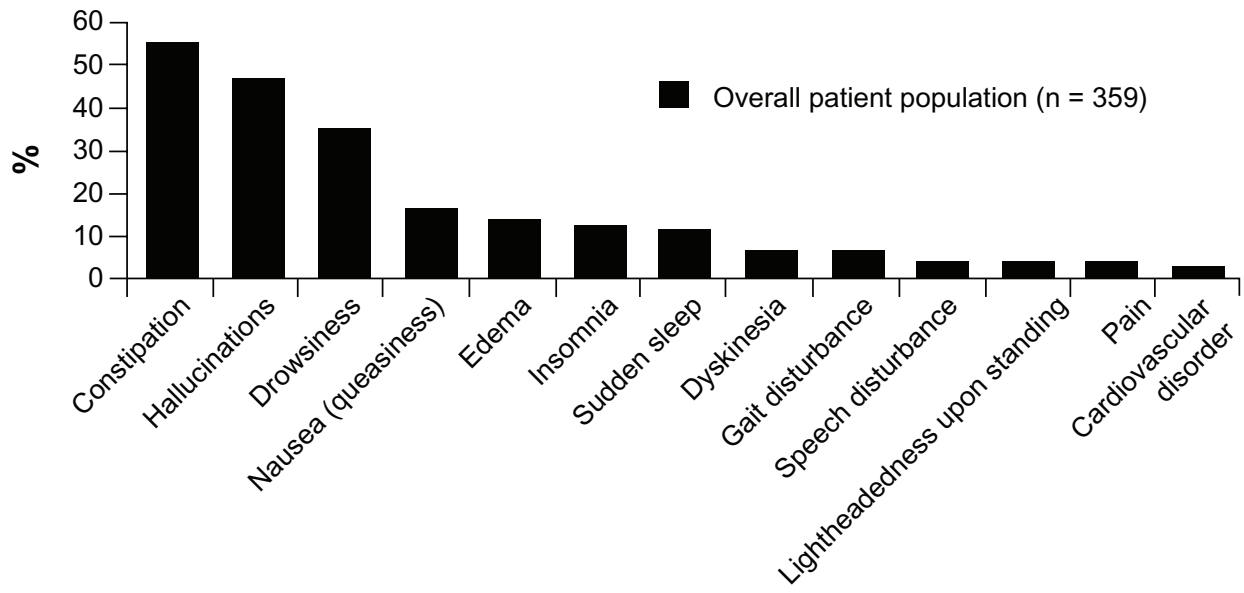

Figure 3 Adverse effects of antiparkinsonian medication that concerned patients in the Japanese survey. Note: Patients who did not include a specific response were excluded from this analysis. 
Table 2 Reasons patients in the US thought they were switched to levodopa-carbidopa, and why they were concerned about taking levodopa-carbidopa

\begin{tabular}{ll}
\hline Perceived reasons for being switched to LC & Patients (\%) \\
My PD symptoms were getting progressively worse & 55 \\
My PD symptoms did not get worse, but I did not & 16 \\
get good symptom control with previous treatments & \\
I do not know/my doctor recommended it & 15 \\
I could not tolerate the side effects of previous & 10 \\
treatments & \\
Other reason & 5 \\
Concerns about taking LC & Patients (\%) \\
$\begin{array}{l}\text { Long-term side effects of LC, such as dyskinesias } \\
\text { (uncontrolled movements, wiggles) }\end{array}$ & 52 \\
$\begin{array}{l}\text { Benefits may begin to wear off sooner than desired } \\
\text { An indication that my PD might have advanced } \\
\text { to a more severe stage }\end{array}$ & 49 \\
Immediate side effects of LC, such as nausea & 46 \\
and vomiting & 34 \\
Fear that LC might make my PD worse & \\
Being able to afford LC & 23 \\
\hline
\end{tabular}

Notes: aRespondents who were not initiated on levodopa $(n=110)$; patients who did not provide a specific response were excluded from this analysis. Abbreviations: LC, levodopa-carbidopa; PD, Parkinson's disease.

Society of Japan conference held in Kyoto, the most commonly used daily dose of levodopa ranged from $300 \mathrm{mg}$ to $400 \mathrm{mg} .{ }^{8}$ For many Japanese providers, the highest daily dose of levodopa was $300 \mathrm{mg}$, even for patients with advanced PD. Consistent with this, about $17 \%$ of patients who were interviewed stated that they had been informed by their physician that their medication could no longer be increased, despite the suggestion of increasing motor disability.

\section{Discussion}

Despite being the most effective treatment for PD, the higher possibility of motor complications associated with levodopa may result in potential underdosing. ${ }^{5}$ Although dyskinesias are often regarded as one of the most important complications of levodopa therapy, ${ }^{9}$ this project suggests that dyskinesias were not a primary concern for patients surveyed in either the US or Japan. In the US, patients were more concerned about wearing-off, whereas other adverse effects, such as hallucinations, were of greater concern to Japanese patients. Interestingly, Japanese patients who had not yet experienced dyskinesia were more concerned about this adverse effect than those with a prior history of dyskinesia, possibly due to concern regarding the mental burden and hardship of the condition. Although primary care providers in the US recognized the importance of wearing-off, specialists considered dyskinesias to be of equal, if not greater, concern for patients. This suggests that patient concerns about dyskinesia may, in some cases, be overestimated by physicians, and may cause some hesitation when prescribing levodopa.

Patient perspectives on treatment options are, among other things, influenced by disease stage, symptom severity, and experience of adverse effects. Understanding patient attitudes towards PD therapies and the associated complications may help physicians devise individualized treatment strategies. There is currently a multitude of therapeutic options for patients with PD, and individual benefit varies significantly among patients. The benefit of efficient communication between the patient and the doctor in any culture cannot be overestimated, particularly when individualizing treatment. However, improved patient education and awareness is paramount for effective patient-physician communication. Patients need to understand the symptoms of $\mathrm{PD}$, and be aware of the implications of certain therapies in order to be familiar with signs of disease progression or treatment complications.

The results of our US survey highlight a further discrepancy between physicians and patients as to reasons for initiation of levodopa-carbidopa therapy: while the majority of patients believed levodopa-carbidopa therapy was initiated because of progressive worsening of PD symptoms, $50 \%$ of family physicians and nearly a third of specialists initiated levodopa-carbidopa therapy at diagnosis. Furthermore, more than half of the US patients said they were at least somewhat concerned about taking levodopa-carbidopa, as a result of information obtained on the Internet or from physicians.

It is interesting to note that while US patients were most concerned about long-term side effects of their medication, such as dyskinesia and wearing-off, Japanese patients worried more about experiencing hallucinations. This is possibly due to the fact that the majority of Japanese patients in this study received dopamine agonist therapy. Hallucinations are more likely to occur with dopamine agonists than with levodopa, ${ }^{6}$ and, in Japan, it is common clinical practice for patients with PD to be initiated on low-dose levodopa combined with dopamine agonists or amantadine. The higher use of dopamine agonists in Japan is also reflected in Japanese clinical trials compared with those conducted in the West. ${ }^{10,11}$ Studies have reported a higher incidence of hallucinations in Japanese patients compared with Western patients, which is attributable to the higher doses of dopamine agonists used in the Japanese PD population. ${ }^{12-14}$ Therefore, because hallucinations can impact on the quality of life of both patients and their caregivers, ${ }^{15}$ it would seem pertinent for physicians in Japan to know how to avoid these adverse 
effects and how to manage drug-induced psychotic symptoms should they arise. ${ }^{15}$

Regarding attitudes towards drug intake and dose increases, it is noteworthy that while almost all US physicians believe patients would rather reduce their pill burden, US patients themselves consider their biggest challenge to be wearing-off. In contrast, patients in Japan would rather increase their dose or dosing frequency in order to ameliorate their symptoms. Indeed, patients in Japan expressed a preference for obtaining symptomatic relief, even if that required an increase in medication dosing. This observation is strengthened further by the fact that this preference for symptomatic relief was similar between patients with or without wearing-off. In addition, one major discrepancy between patients and physicians, in both the US and Japan, related to dose increases. In the US, patients feared wearing-off, yet physicians were under the impression that patients wanted to restrict medication intake; However, in Japan, patients seek symptomatic relief, even if that results in an increase in medication. Despite this, the conference survey results indicated that physicians in Japan are reluctant to increase doses. This is supported by results of Japanese studies advocating the use of low doses of levodopa to avoid the development of motor complications. ${ }^{12}$ Therefore, the findings demonstrate a need for improved communication between doctors and patients in both countries regarding dose increases, taking into account patient perspectives of adverse effects.

The difference between patient perspectives among Japanese and US patients is likely to stem from differences in medical practice for the management of PD. However, the underlying reasons for this difference are unclear. One possibility is that availability of certain antiparkinsonian therapeutic agents in the two countries may differ. For example, the triple combination therapy levodopa/carbidopa/entacapone is not yet available in Japan, whereas certain dopamine agonists, such as talipexole and droxidopa, are only marketed in Japan. Monotherapy with selegiline is not covered by Japanese health insurance, because it has not been approved by the local authorities. ${ }^{16}$ Another possible difference that may influence decisions on therapy is the cost of the drug in the respective countries. In Japan, the cost of antiparkinsonian therapies is largely covered by government-funded Japanese health insurance. In the US, the cost of the drugs depends on the specific health care insurance scheme in which the patient in enrolled. However, given that the cost of levodopa is much lower than that of dopamine agonists, it is unlikely to play a significant role in determining whether to introduce levodopa or whether increases in its dosage or dosing frequency are required. ${ }^{17}$
In fact, in Japan, the cost of levodopa and dopamine agonists will be covered by national insurance (at least for patients with Hoehn and Yahr stage III or higher), and is unlikely to be a driving factor for the choice of therapy used in this region. Therefore, the reason why the doses of levodopa used in Japan tend to be lower than in the West is unclear. Results from a retrospective study based at the Sapporo Azabu Neurosurgical Hospital in Japan suggested that lower doses of levodopa may be sufficient to achieve symptom control and may reduce or delay the appearance of motor complications compared with the higher doses of levodopa required to achieve symptom control in multinational, randomized, controlled trials. ${ }^{12,18-20}$ The authors of the former study proposed that Japanese patients with PD may respond better to levodopa compared with their Caucasian counterparts, and speculated that variations in genetic background, pharmacokinetics, and lifestyle choices may contribute to this difference. ${ }^{12}$ It is also likely that physicians in Japan are concerned about dyskinesias, which tend to be associated with levodopa, and try as much as possible to avoid the development of this complication. ${ }^{12}$ Finally, a long-term anti-levodopa campaign, which focused on the potential neurotoxicity of levodopa, and interpretation of the 2002 Japanese practice guidelines for PD, may play a role in influencing attitudes in Japan. ${ }^{16,21}$ Although, the seminal ELLDOPA (Earlier vs Later L-DOPA) study of levodopa in early PD patients has dispelled the notion that levodopa is neurotoxic, ${ }^{22}$ concerns may still resonate with many Japanese physicians. However, underdosing with levodopa can be associated with a reduction in symptom control and, consequently, may impact patient quality of life. ${ }^{5}$ In addition, the observations that dyskinesia is not a major concern for patients in this study and that patients in Japan prefer increasing the dose of medication to improve symptom control, suggests that physicians should not limit the dose of levodopa to avoid the development of dyskinesia.

This study set out to elucidate the perspectives of patients towards PD and antiparkinsonian therapy and to understand whether such views and concerns differ between patients in the US and those in Japan. Although in some cases (eg, those with cognitive or physical difficulties), the patient's caregiver may have completed the Japanese survey on behalf of the patient, this is unlikely to have affected the study results significantly. However, it should be noted that the way in which the two surveys were conducted varied slightly, and the results between the two countries may not be directly comparable. As such, some caution must be exercised when interpreting these results. Nevertheless, the study highlights some interesting similarities and differences between the 
two populations, as well as differences between patient and physician perspectives in both countries.

\section{Conclusion}

In conclusion, this study suggests that patient perceptions about PD therapy may differ from the views of their physicians. Heightened understanding of patient concerns and attitudes towards PD treatments and their associated complications may help physicians to individualize optimal treatment strategies. Improving patient education and awareness about PD and medical therapy will be instrumental in enhancing patient-physician communication and, consequently, patient care and treatment outcomes.

\section{Disclosure}

Funding for these studies was provided by Novartis Pharma KK and Novartis Pharmaceuticals Corporation. Nadia Hashash provided editorial assistance which was funded by Novartis Pharmaceuticals Corporation and Orion Pharmaceuticals Corporation. NH, KF, TK, and MS have received sponsorship funding from Novartis Pharma KK, Novartis Pharmaceuticals Corporation, and Orion Pharmaceuticals Corporation; consultancy fees from the Japan Management Association and Novartis; and research grants from Novartis Pharma KK. MM has received sponsorship funding from Novartis Pharma KK, Novartis Pharmaceuticals Corporation, and Orion Pharmaceuticals Corporation; and consultancy fees from the Japan Management Association. MS has received grant support from Ceregene, IMPAX, the Michael J Fox Foundation, Neuraltus, Novartis Pharmaceuticals Corporation, Parkinson Study Group, and ScheringPlough; consultancy fees from Allergan, General Electric, Novartis, Orion Osmotica, and Schering-Plough; and serves on protocol steering committees for EMD Serono, Novartis Pharmaceuticals Corporation, Allergan, and Teva. This work has previously been presented as a poster (16.04) at the 2nd World Parkinson Congress, September 28 to October 1, 2010, Glasgow, UK.

\section{References}

1. Stewart MA. Effective physician-patient communication and health outcomes: a review. Can Med Assoc J. 1995;152(9):1423-1433.

2. Shortus T, Rose V, Comino E, Zwar N. Patients' views on chronic illness and its care in general practice. Aust Fam Physician. 2005;34(5):397-399.
3. Osman LM, Hyland ME. Patient needs and medication styles in COPD Eur Respir Rev. 2005;14(96):89-92.

4. Sirey JA, Bruce ML, Alexopoulos GS, et al. Perceived stigma as a predictor of treatment discontinuation in young and older outpatients with depression. Am J Psychiatry. 2001;158(3):479-481.

5. Poewe W, Antonini A, Zijlmans JCM, Burkhard PR, Vingerhoets F. Levodopa in the treatment of Parkinson's disease: an old drug still going strong. Clin Interv Aging. 2010;5:229-238.

6. Antonini A, Tolosa E, Mizuno Y, Yamamoto M, Poewe WH. A reassessment of risks and benefits of dopamine agonists in Parkinson's disease. Lancet Neurol. 2009;8(10):929-937.

7. Hoehn HM, Yahr MD. Parkinsonism: onset, progression, and mortality Neurology. 1967;17(5):427-442.

8. Novartis Pharma K.K. Data on file. 2008.

9. Encarnacion EV, Hauser RA. Levodopa-induced dyskinesias in Parkinson's disease: etiology, impact on quality of life, and treatments. Eur Neurol. 2008;60(2):57-66.

10. Rinne UK. Entacapone an effective levodopa enhancer: experience from clinical studies. Presented at the 32nd Scandinavian Congress of Neurology, Oulu, Finland, June 10-13, 1998.

11. Ooba N, Yamaguchi T, Kubota K. The impact in Japan of regulatory action on prescribing of dopamine receptor agonists: analysis of a claims database between 2005 and 2008. Drug Saf. 2011;34(4):329-338.

12. Kitagawa M, Tashiro K. Low-dose levodopa therapy in Japanese patients with Parkinson's disease: a retrospective study. Intern Med. 2005;44(9):939-943.

13. Aarsland D, Larsen JP, Cummins JL, Laake K. Prevalence and clinical correlates of psychotic symptoms in Parkinson disease: a communitybased study. Arch Neurol. 1999;56(5):595-601.

14. Holroyd S, Currie L, Wooten GF. Prospective study of hallucinations and delusions in Parkinson's disease. J Neurol Neurosurg Psychiatry. 2001;70(6):734-738.

15. Kuzuhara S. Drug-induced psychotic symptoms in Parkinson's disease. Problems, management and dilemma. J Neurol. 2001;248 Suppl 3: III28-III31.

16. Kondo T. Treatment of Parkinson's disease in Japan. Parkinsonism Relat Disord. 2000;7(1):71-77.

17. Factor SA, Weiner WJ. Parkinson's Disease: Diagnosis and Clinical Management: New York, NY: Demos Medical Publishing LLC; 2008.

18. Larsen JP, Boas J, Erdal JE. Does selegiline modify the progression of early Parkinson's disease? Results from a five-year study. The Norwegian-Danish Study Group. Eur J Neurol. 1999;6(5): 539-547.

19. Holloway RG, Shoulson I, Fahn S, et al. Pramipexole vs levodopa as initial treatment for Parkinson disease: a 4-year randomized controlled trial. Arch Neurol. 2004;61(7):1044-1053.

20. Bracco F, Battaglia A, Chouza C, et al. The long-acting dopamine receptor agonist cabergoline in early Parkinson's disease: final results of a 5-year, double-blind, levodopa-controlled study. CNS Drugs. 2004;18(11):733-746.

21. Shibuya M, Hirose G, Sakai F, et al. Guidelines for the treatment of Parkinson's disease. Clin Neurol. 2002;42(5):421-494.

22. Fahn S, Oakes D, Shoulson I, et al. Levodopa and the progression of Parkinson's disease. N Engl J Med. 2004;351(24):2498-2508. 


\section{Publish your work in this journal}

Patient Related Outcome Measures is an international, peer-reviewed, open access journal focusing on treatment outcomes specifically relevant to patients. All aspects of patient care are addressed within the journal and practitioners from all disciplines are invited to submit their work as well as healthcare researchers and patient support groups. Areas covered will

include: Quality of life scores; Patient satisfaction audits; Treatment outcomes that focus on the patient; Research into improving patient outcomes; Hypotheses of interventions to improve outcomes; Short communications that illustrate improved outcomes; Case reports or series that show an improved patient experience; Patient journey descriptions or research.

Submit your manuscript here: http://www.dovepress.com/patient-related-outcome-measures-journal 